A) Check for updates

Cite this: Org. Biomol. Chem., 2019, 17, 2315

Received 29th November 2018,

Accepted 28th January 2019

DOI: $10.1039 / c 80 b 02977 f$

rsc.li/obc

\section{An atom efficient synthesis of tamoxifen $\dagger$}

\author{
Dorus Heijnen, $\$$ Milan van Zuijlen, + Filippo Tosił and Ben L. Feringa (D) *
}

The direct carbolithiation of diphenylacetylenes and their crosscoupling procedure taking advantage of the intermediate alkenyllithium reagents are presented. By employing our recently discovered highly active palladium nanoparticle based catalyst, we were able to couple an alkenyllithium reagent with a high (Z/E) selectivity $(10: 1)$ and good yield to give the breast cancer drug tamoxifen in just 2 steps from commercially available starting materials and with excellent atom economy and reaction mass efficiency.

The continuous improvement of synthetic routes towards medicinally relevant and/or biologically active compounds has drawn the attention of synthetic chemists for decades. ${ }^{1}$ In order to reduce waste and increase yields and cost efficiency or to simplify the procedures towards relevant structures, transition metal catalysis has been a game changer in the field. ${ }^{2}$ Since the emergence of the Suzuki (B), Stille (Sn), Negishi (Zn) and Hiyama-Denmark ( $\mathrm{Si}$ ) reactions, the trend in the crosscoupling methodology ${ }^{3}$ has been to transmetallate highly polar (but straightforward to synthesize) organometallic reagents ( $\mathrm{RMgX}, \mathrm{RLi})$ to softer nucleophiles in order to gain stability and functional group tolerance and reduce the overall sensitivity of the reaction. Despite their major role in our modern synthetic toolbox, the drawbacks of these additional synthetic steps are longer reaction times, the production of stoichiometric (sometimes toxic) waste, and a decrease in cost efficiency. ${ }^{4}$ Nonetheless, the direct coupling of organometallic reagents arising from a deprotonation or umpolung reaction has shown great advances in recent years. ${ }^{2,5}$ Since these reagents have an intrinsically higher reactivity, the corresponding cross-coupling reactions generally require a shorter reaction time, and can be performed at significantly lower temperatures. ${ }^{4}$ As part of our effort to expand the synthetic

Stratingh Institute for Chemistry, University of Groningen, Nijenborgh 4,

9747 AG, Groningen, The Netherlands.E-mail: b.l.feringa@rug.nl

$\dagger$ Electronic supplementary information (ESI) available. See DOI: 10.1039/ c8ob02977f

\$These authors made equal contribution to this work. application of our recently reported organolithium cross-coupling reactions, ${ }^{5 d_{2}, g, i-k}$ we envisioned the direct carbolithationcross coupling to be a very valuable alternative. The carbolithiation of (diphenyl)acetylenes has been well-studied and has led to several useful applications in the field of synthetic organic chemistry. ${ }^{6-10}$ The quenching of the formed nucleophilic $\mathrm{sp}^{2}$ vinyllithium reagent with an electrophile provides a direct approach to substituted diarylalkenes (stilbenes). Transmetallation of such anions to magnesium, boron, zinc or even aluminum yields an active cross-coupling partner, but drastically lowers the atom economy and $E$-factor. ${ }^{11,12}$ The direct cross-coupling of the formed organolithium reagent is therefore a highly desired synthetic shortcut, but remains unreported to the best of our knowledge. Tetrasubstituted alkenes and triphenylethylenes in particular, i.e. tamoxifen, make up a class of highly potent and valuable drugs with (potential) application in the treatment of a variety of conditions, including (breast) cancer, dyspareunia and osteoporosis (Fig. 1). ${ }^{13}$ Structural variations in the triphenylethylene scaffold are found in the alkyl-ether substituent (mostly consisting of an amine, shown in blue) and para phenylene functionality (shown in red) as well as in the alkyl fragment (shown in green) on the remaining alkene position.
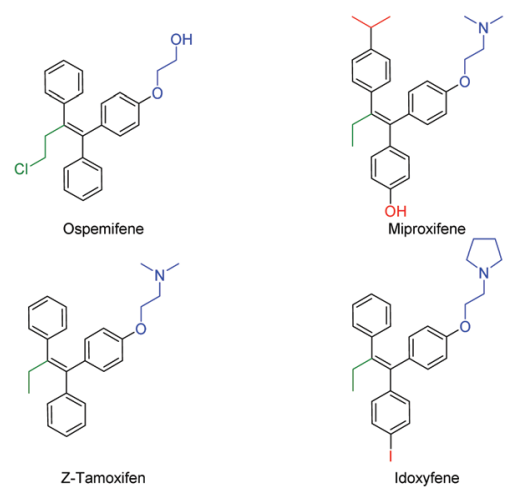

Fig. 1 Examples of members of the triphenylethylene family of drugs. 


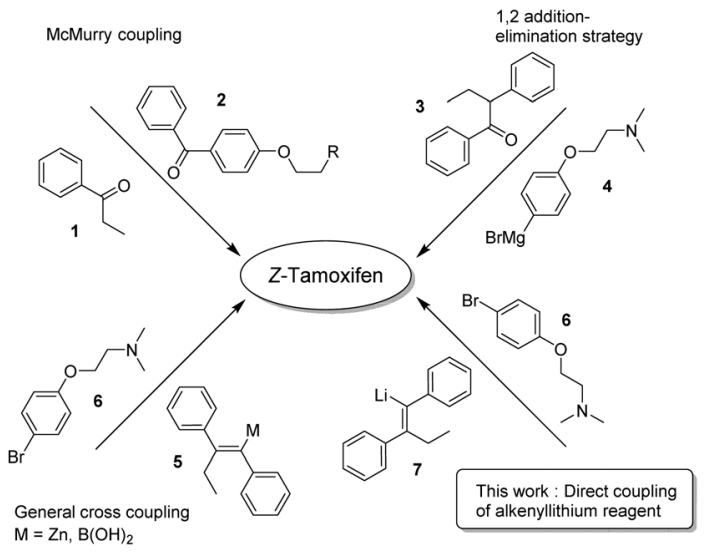

Fig. 2 Synthetic approaches to (Z)-tamoxifen.

Because of its medicinal importance, a plethora of syntheses have been described for (Z)-tamoxifen (Fig. 2). ${ }^{15-37}$ McMurry coupling of two ketones is a well-established method for the synthesis of (hindered) alkenes, and as such has proven to be capable of constructing the alkene fragment in tamoxifen with reagents 1 and $2 .{ }^{21}$ Alternatively, 1,2-addition to ketone 3 with Grignard reagent $\mathbf{4}$ followed by elimination yielding an alkene is a valuable option, however it is common that both isomers $(E / Z)$ are isolated via this approach. ${ }^{19,24}$ The transmetallation of the lithium intermediate that is the product of carbolithation of the corresponding acetylene yields the alkenylboronic acid/ester, or organozinc reagent $5 .^{20,22}$ The crosscoupling of these reagents with bromide 6 provides a viable route towards the final drug. The transmetallation, however, generates extra synthetic steps and/or stoichiometric waste. We therefore reasoned that the direct coupling of the alkenyllithium reagent 7 , which is obtained upon carbolithiation of diphenylacetylene, would be an important atom efficient alternative to these methods.

In order to optimize the sequential synthetic steps, the carbolithiation of acetylene $\mathbf{8}$ was optimized separately, and the product 10 resulting from $\mathrm{MeI}$ quenching was subjected to GC-MS analysis (Table 1). With a high selectivity for the $(Z)$-alkenes for several solvents and solvent mixtures being investigated, we tried to avoid the use of THF (Table 1, entry 1) due to expected difficulties in the cross-coupling step arising from unwanted side reactions, such as lithium-halogen exchange. ${ }^{5 b-g, i-l}$ However, toluene/TMEDA mixtures (entry 2) or other ethereal solvents (entries 3-5) did not prove equally efficient as the reaction medium compared to THF due to a lower extent of lithiation and an increased amount of the $(E)$-alkene. Attempts to minimize waste production by neat carbolithiation (except for the solvent of the $n$-BuLi solution) resulted in mere recovery of the starting material (entry 6). Reducing the amount of THF by using a THF-toluene mixture resulted in incomplete conversion to the carbolithiated intermediate (entry 7). Despite the attempts to omit THF as the solvent, we found significantly better results for the carbolithiation in its presence, and therefore decided to use it as the solvent for further optimization.
Table 1 Optimization of carbolithiation of 8

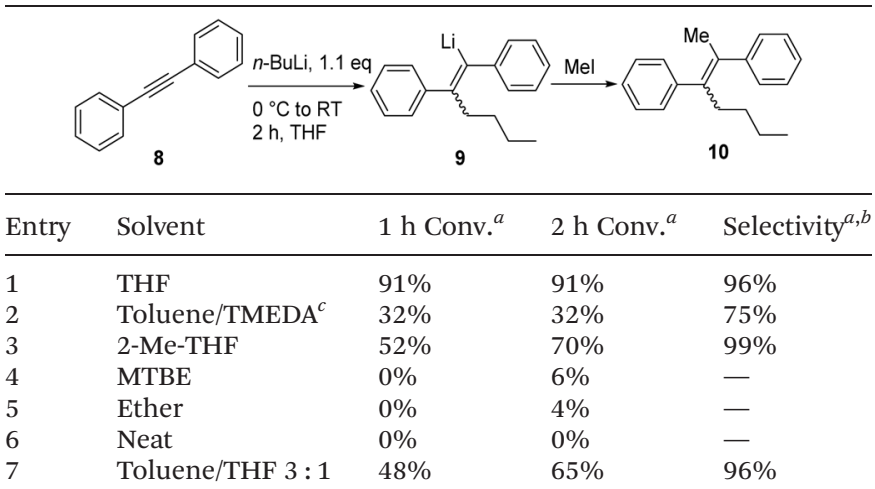

Reaction conditions: 1.1 eq. of $n$-BuLi were added dropwise to a stirred $0.9 \mathrm{M}$ solution of 8 in $\mathrm{THF}$ at $0{ }^{\circ} \mathrm{C}$. At the end of the addition, the reaction mixture was quickly allowed to warm to room temperature and left to react for $2 \mathrm{~h}$ before 1 eq. of MeI was added. ${ }^{a}$ As determined by GC-MS analysis after MeI quenching. ${ }^{b}(Z / E)$ selectivity determined by GC-MS analysis. ${ }^{c} 1$ eq. of TMEDA.

Having established the optimized conditions for the carbolithiation, the cross-coupling with 1-bromonaphthalene provided the test reaction in pursuit of the best catalyst. Our oxygen activated palladium nanoparticle catalytic system ${ }^{5 k}$ proved to be highly active in the coupling of alkenyllithium 9 and arylbromides (Table 2, entry 1 ), being only slightly outperformed by the commercial Pd-PEPPSI-Ipent complex (entry 4).

Nickel and palladium bisphosphine complexes (Pd-dppf and Ni-dppp) did not show conversion to the desired product

Table 2 Optimization of cross-coupling of 9 with 1-bromonaphthalene

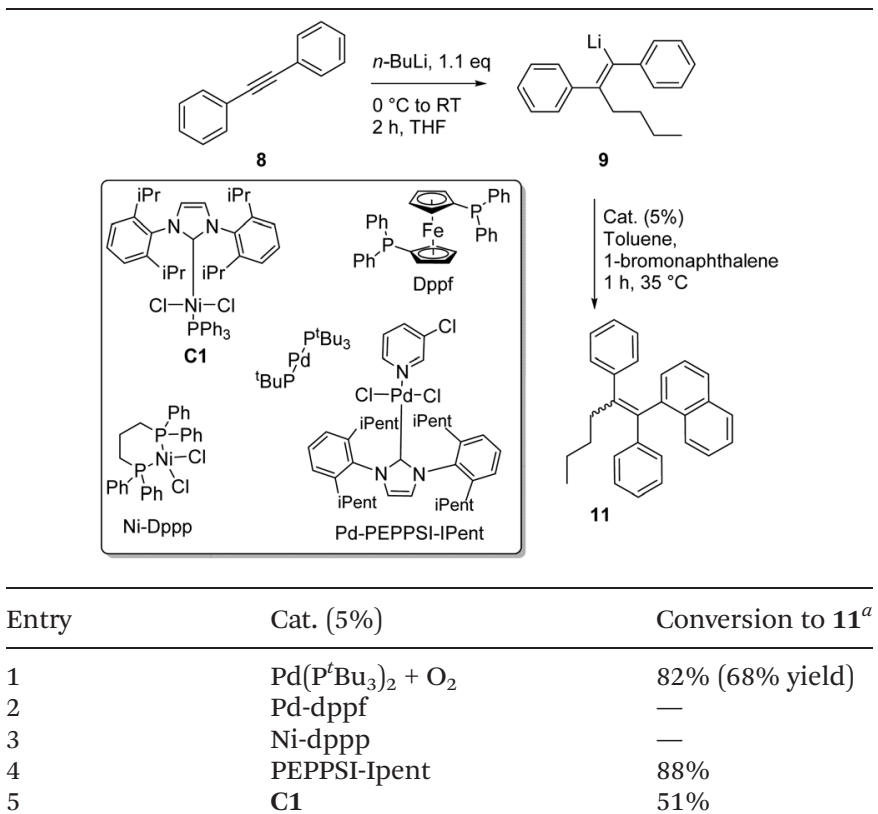

Reaction conditions: 2 eq. of alkenyllithium reagent were added over $20 \mathrm{~min}$ to a stirred solution of arylbromide and (pre-oxidized) catalyst in toluene at $35^{\circ} \mathrm{C} .{ }^{a}$ As determined by GCMS analysis. $(Z / E)>9: 1$. 
(entries 2 and 3), but the nickel carbene complex $\mathbf{C} 1^{5 j}$ did provide the triarylethylene target 11 (entry 5), albeit in a reduced yield (for further attempts to use different electrophiles, see the ESI $\dagger$ ). In order to see if this cross-coupling methodology could be applicable for the cross-coupling of other arylbromides, we decided to test a few further substrates (Table 3). The cross-coupling methodology proceeds smoothly with both electron rich arylbromides $(\mathrm{R}=\mathrm{Me}$, OMe, entries 1 and 2) and electron poor substrates ( $\mathrm{R}=\mathrm{CF}_{3}$, entry 3$)$.

Having tested a small variety of catalysts for the cross coupling with different substrates, the optimized conditions were employed to synthesize the desired pharmaceutical (Z)-tamoxifen via our new methodology. Changing the nucleophile for the acetylene carbolithiation from $n$-butyllithium to ethyllithium gave identical results albeit with a slightly longer reaction time. We were pleased to see that the oxygenated Pd $\left(\mathrm{P}^{t} \mathrm{Bu}_{3}\right)_{2}$ catalyst gave $(Z)$-tamoxifen in only a slightly lower yield than with the naphthalene test substrate, but with very good $(Z / E)$ selectivity (Table 4 , entry $1,(Z / E)>9: 1)$. On pursuing a cheaper catalyst, with a more abundant metal, the attempted nickel complex gave only small amounts of the desired product (entry 2).

Much to our surprise, our "working horse" catalyst PdPEPPSI Ipent ${ }^{5 g, i}$ was completely inactive in the cross-coupling with the bromophenyl-aminoether electrophile 6 (Table 4, entry 3), whereas it proved to be the most active catalyst with the previously tested 1-bromonaphthalene electrophile (Table 2, entry 4). The chelating effect of the amino-ether moiety onto the metal center, overcrowding the palladium, might play a role making the catalyst inactive. Other dimeric palladium phosphine complexes, which have recently been shown to be active in related cross-coupling reactions, were also tested, ${ }^{5 h, k}$ and were found to have very similar reactivity compared to the catalyst used in entry 1 . Being the cheapest of the three related structures (entries 1, 5 and 6), we decided to proceed our investigation with the bis(tri-tert-butylphosphine) palladium complex. The results of further optimization are shown in Table 5. Varying the temperature did not lead to an

Table 3 Reaction scope of cross-coupling of 9 with different aryl bromides

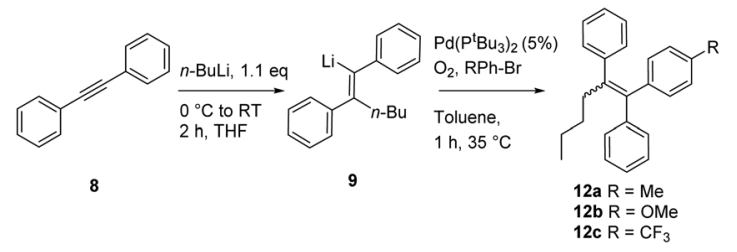

\begin{tabular}{llll}
\hline Entry & $\mathrm{R}$ & Product & Yield of $\mathbf{1 2}^{a}$ \\
\hline 1 & $\mathrm{Me}$ & $\mathbf{1 2 a}$ & $56 \%$ \\
2 & $\mathrm{OMe}$ & $\mathbf{1 2 b}$ & $51 \%$ \\
3 & $\mathrm{CF}_{3}$ & $\mathbf{1 2 c}$ & $68 \%$
\end{tabular}

Reaction conditions: 2 eq. of alkenyllithium reagent were added over $20 \mathrm{~min}$ to a stirred solution of arylbromide and (pre-oxidized) catalyst in toluene at $35^{\circ} \mathrm{C} .^{a}$ Isolated yield after column chromatography.
Table 4 Synthesis of tamoxifen: catalyst screening

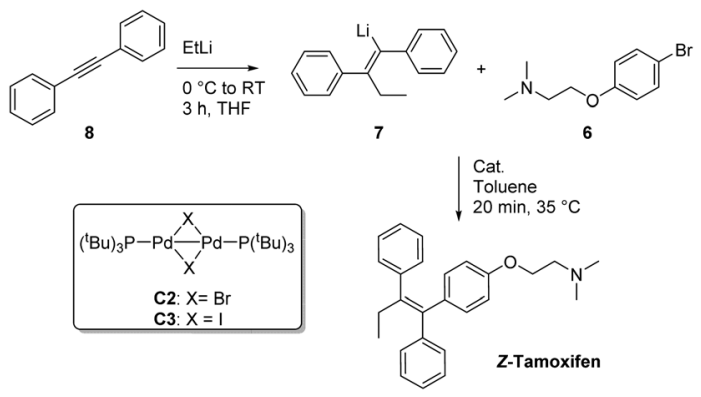

\begin{tabular}{lll}
\hline Entry & Cat. $(5 \%)$ & NMR yield $^{a}$ \\
\hline 1 & $\mathrm{Pd}^{t}\left(\mathrm{P}^{t} \mathrm{Bu}_{3}\right)_{2}+\mathrm{O}_{2}$ & $50-65 \%{ }^{b}$ \\
2 & $\mathrm{C} 1$ & $17 \%$ \\
3 & $\mathrm{PEPPSI}-\mathrm{IPent}$ & $0 \%$ \\
4 & $\mathrm{Pd}_{2} \mathrm{dba}_{3} /$ Xphos & $36 \%$ \\
5 & $\mathrm{C2}^{c}$ & $60 \%$ \\
6 & $\mathrm{C3}^{c}$ & $59 \%$
\end{tabular}

Reaction conditions: 2 eq. of alkenyllithium reagent were added over $20 \mathrm{~min}$ to a stirred solution of arylbromide and (pre-oxidized) catalyst in toluene at $35{ }^{\circ} \mathrm{C}$. ${ }^{a}$ Yield determined by ${ }^{1} \mathrm{H}$-NMR with 1,1,2,2-tetrachloroethane as the internal standard, accompanied by $<10 \%$ of $(E)$ tamoxifen relative to (Z)-tamoxifen. ${ }^{b}$ Isolated yield up to $57 \%$. ${ }^{c} 2.5 \mathrm{~mol} \%$ of the Pd dimer was used.

Table 5 Optimization of the carbolithiation-cross coupling sequence ${ }^{14}$

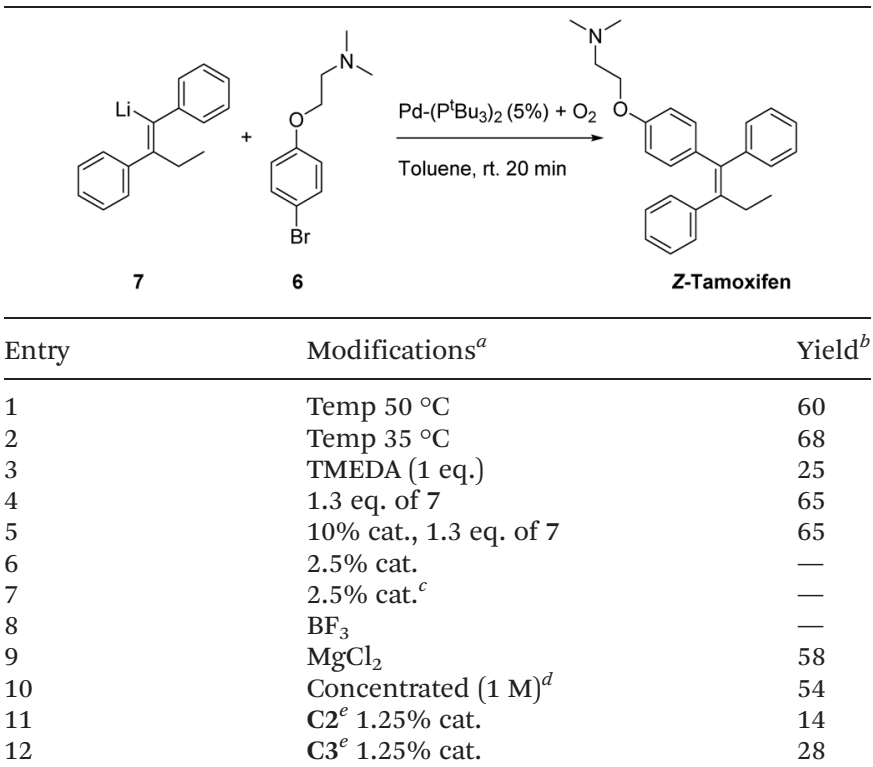

${ }^{a}$ Reaction conditions: 2 eq. of alkenyllithium reagent were added over $20 \mathrm{~min}$ to a stirred solution of arylbromide $(2 \mathrm{mmol})$ and pre-oxidized catalyst in toluene $(2 \mathrm{ml})$ at room temperature. ${ }^{b}$ Yield determined by ${ }^{1} \mathrm{H}$-NMR with 1,1,2,2-tetrachloroethane as the internal standard. ${ }^{c}$ Different batches of catalyst. ${ }^{d}$ Initial concentration of arylbromide. ${ }^{e}$ The complexes $\mathbf{C} \mathbf{2}$ and $\mathbf{C} \mathbf{3}$ were used with the same Pd concentration as entries 6 and 7.

increased yield (entries 1 and 2) provided the temperature was kept above $30^{\circ} \mathrm{C}$, below which no conversion was observed. In an attempt to dissociate potential aggregates and activate the 
organolithium reagent, TMEDA was added, but this resulted in a sharp decline in yield (entry 3 ). The excess of organolithium reagent could be lowered to 1.3 equivalents without a significant loss in yield (entry 4). Further lowering of the catalyst loading (2.5 mol\%) led to an inactive system, with no product formed (entries 6 and 7). This complete deactivation of the catalyst at $2.5 \mathrm{~mol} \%$ has not been observed before, and it is potentially attributed to the chelating effect of the aminoether moiety that is present in the substrate. An attempt to prevent the chelating effect of the aminoether side chain by means of the addition of Lewis acids such as $\mathrm{BF}_{3}$ or $\mathrm{MgCl}_{2}$ did not prove beneficial for the reaction (entries 8 and 9). To minimize waste caused by the solvent, the reaction was performed in a minimal amount of solvent, with a $1 \mathrm{M}$ concentration, which only led to a slight decrease in yield (entry 10). Lower loadings (1.25 mol\%) of complexes C2 and C3 provided better conversion than our catalyst of choice at those concentrations (entries 6, 11 and 12), but still providing lower overall yields (14\% and $28 \%$, respectively).

Having a setup that produces this pharmaceutical compound in good yield and with minimal waste (LiBr being the only stoichiometric waste in the last step), we compared our procedure with other (recent) reported syntheses of tamoxifen ${ }^{6,15-37}$ focusing on atom economy and Reaction Mass Efficiency (RME). ${ }^{12 b}$ Fig. 3 shows a large range in atom economy (shown in blue) between different reported syntheses of tamoxifen. The method described by Larock in $2005^{37}$ is the closest to the reported route in this work in terms of atom economy (48 vs. 67\%), but due to a large excess of some reagents, it scores much lower on RME (shown in red).

With our current setup, by employing commercially available starting materials, a total atom economy of 0.67 is achieved, and the resulting RME (22\%) is almost twice as high as that of the runner-up (Knochel, 1997, 11\%). ${ }^{20}$ We believe that the currently reported methodology presents additional relevant advantages, since $\mathrm{LiBr}, \mathrm{NaCl}$ and $\mathrm{HCl}$ are the only stoichiometrically produced waste sources, and the reaction can be performed at a slightly elevated temperature in a minimal amount of solvent.

To establish the optimal isolation method, the synthesized $(Z)$-tamoxifen was purified by means of crystallization, extrac-

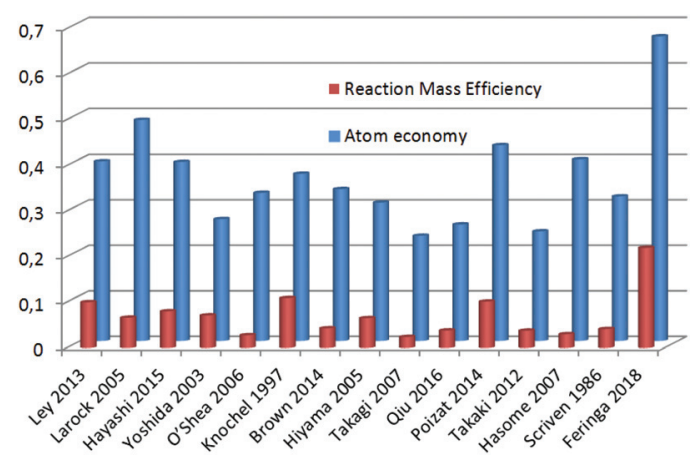

Fig. 3 Atom economy and RME comparison for each reported tamoxifen synthesis.

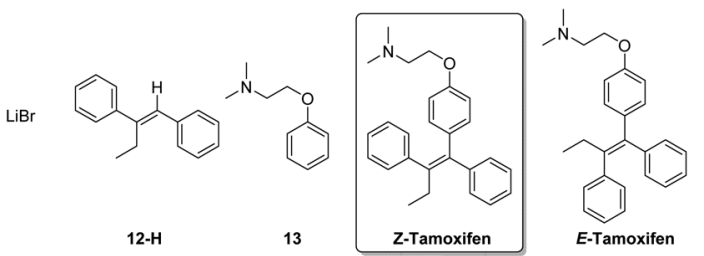

Fig. 4 (Z)-Tamoxifen and side products.

tion, column chromatography and distillation. The excess organolithium reagent (protonated after reaction quenching, 12-H) and the formed lithium bromide pose no difficulty in the separation from the product (Fig. 4). Acid-base extraction and column chromatography were both suitable means to achieve purification.

The remaining impurity of dehalogenated starting material 13 exhibits near identical behavior compared to the product, but flash chromatography was able to yield the pure $(E / Z)$ tamoxifen mixture. If desired, RP-preparative HPLC in water/ acetonitrile/TFA effectively separates the $(E)$ from the $(Z)$ isomer of the product.

In conclusion, the carbolithiation of diphenylacetylene and the consecutive cross-coupling with the appropriate 4-bromodimethylamine-ethylether (6) yields $(Z)$-tamoxifen with a good $(Z / E)$ selectivity $(10: 1)$ and with yields up to $65 \%$. The reaction mixture was purified by flash chromatography to obtain the pure $(E / Z)$-tamoxifen mixture. Further optimization could lead to a lowering of the catalyst loading and suppression of the lithium halogen exchange or $E-Z$ isomerisation that lead to the undesired side products which have proven to be a challenge in the purification of this pharmaceutical. The method distinguishes itself from previously reported syntheses by its high atom economy, reaction mass efficiency, non-toxic waste production, step count and ease of reaction setup. The organolithium cross-coupling is also an attractive strategy for the coupling of less reactive electrophiles (chlorides, fluorides and ethers) $^{5 j}$ and future studies might further enhance the efficiency towards triarylethylenes and related products.

\section{Conflicts of interest}

There are no conflicts of interest to declare.

\section{Acknowledgements}

B. L. F. acknowledges financial support from the Netherlands Organisation for Scientific Research, the European Research Council (ERC Advanced Grant 227897), the Royal Netherland Academy of Arts and Sciences (KNAW, Gravitation program 024.601035), and the Ministry of Education, Culture and Science. 


\section{References}

1 (a) X.-F. Wu, P. Anbarasan, H. Neumann and M. Beller, Angew. Chem., Int. Ed., 2010, 49, 9047-9050; (b) B. M. Rosen, K. W. Quasdorf, D. A. Wilson, N. Zhang, A.-M. Resmerita, N. K. Garg and V. Percec, Chem. Rev., 2011, 111, 1346-1416; (c) J. J. Li and E. J. Corey, Total Synthesis of Natural Products: At the Frontiers of Organic Chemistry, Springer, Heidelberg, 2012; (d) L. Guo, C.-C. Hsiao, H. Yue, X. Liu and M. Rueping, ACS Catal., 2016, 6, 4438-4442; (e) M. Tobisu, T. Takahira, T. Morioka and N. Chatani, J. Am. Chem. Soc., 2016, 138, 67116714.

2 (a) N. Miyaura and A. Suzuki, Chem. Rev., 1995, 95, 24572483; (b) F. Diederich and P. J. Stang, Metal-Catalyzed Cross-Coupling Reactions, Wiley-VCH, New York, 1998; (c) R. Martin and S. L. Buchwald, Acc. Chem. Res., 2008, 41, 1461-1473; (d) C. E. I. Knappke and A. J. von Wangelin, Chem. Soc. Rev., 2011, 40, 4948-4962.

3 E. Negishi, Angew. Chem., Int. Ed., 2011, 50, 6738-6764.

4 E. B. Pinxterhuis, M. Giannerini, V. Hornillos and B. L. Feringa, Nat. Commun., 2016, 7, 11698.

5 (a) K. C. Nicolaou, P. G. Bulger and D. Sarlah, Angew. Chem., Int. Ed., 2005, 44, 4442-4489; (b) F. Leroux, M. Schlosser, E. Zohar and I. Marek, The Preparation of Organolithium Reagents and Intermediates in Patai's Chemistry of Functional Groups, 2009; (c) C. C. C. Johansson Seechurn, M. O. Kitching, T. J. Colacot and V. Snieckus, Angew. Chem., Int. Ed., 2012, 51, 5062-5085; (d) V. Hornillos, M. Giannerini, C. Vila, M. Fañanás-Mastral and B. L. Feringa, Org. Lett., 2013, 15, 5114-5117; (e) R. Luisi and V. Capriati, Lithium Compounds in Organic Synthesis, Wiley VCH, Weinheim, 2014; (f) M. Giannerini, M. Fañanás-Mastral and B. L. Feringa, Nat. Chem., 2013, 5, 667-672; (g) L. M. Castelló, V. Hornillos, C. Vila, M. Giannerini, M. Fañanás-Mastral and B. L. Feringa, Org. Lett., 2015, 17, 62-65; (h) M. Aufiero, T. Scattolin, F. Proutiere and F. Schoenebeck, Organometallics, 2015, 34, 5191-5195; (i) J. Buter, D. Heijnen, C. Vila, V. Hornillos, E. Otten, M. Giannerini, A. J. Minnaard and B. L. Feringa, Angew. Chem., Int. Ed., 2016, 55, 3620-3624; (j) D. Heijnen, J.-B. Gualtierotti, V. Hornillos and B. L. Feringa, Chem. Eur. J., 2016, 22, 3991-3995; (k) D. Heijnen, F. Tosi, C. Vila, M. C. A. Stuart, P. H. Elsinga, W. Szymanski and B. L. Feringa, Angew. Chem., Int. Ed., 2017, 56, 3354-3359; (l) M. Busch, M. D. Wodrich and C. Corminboeuf, ACS Catal., 2017, 7, 5643-5653.

6 (a) N. F. McKinley and D. F. O’Shea, J. Org. Chem., 2006, 71, 9552-9555; (b) S. Murahashi, M. Yamamura, K. Yanagisawa, N. Mita and K. Kondo, J. Org. Chem., 1979, 44, 2408-2417.

7 C. Fressigné, A.-L. Girard, M. Durandetti and J. Maddaluno, Angew. Chem., Int. Ed., 2008, 47, 891893.

8 G. Wu, F. E. Cederbaum and E. Negishi, Tetrahedron Lett., 1990, 31, 493-496.
9 C. Fressigné, R. Lhermet, A.-L. Girard, M. Durandetti and J. Maddaluno, J. Org. Chem., 2013, 78, 9659-9669.

10 E. Shirakawa, D. Ikeda, T. Ozawa, S. Watanabe and T. Hayashi, Chem. Commun., 2009, 1885-1887.

11 (a) R. A. Sheldon, Chem. Commun., 2008, 3352-3365; (b) B. H. Lipshutz, N. A. Isley, J. C. Fennewald and E. D. Slack, Angew. Chem., Int. Ed., 2013, 52, 1095210958.

12 (a) B. M. Trost, Angew. Chem., Int. Ed. Engl., 1995, 34, 259281; (b) A. P. Dicks and A. Hent, Green Chemistry Metrics: a Guide to Determining and Evaluating Process Greenness, Springer, 2015.

13 C. Avendano and C. Menendez, Medicinal Chemistry of Anticancer Drugs, Elsevier Science, 2015.

14 For further optimization studies, see the Experimental section.

15 K. Itami, T. Kamei and J. Yoshida, J. Am. Chem. Soc., 2003, 125, 14670-14671.

16 P. E. Tessier, A. J. Penwell, F. E. S. Souza and A. G. Fallis, Org. Lett., 2003, 5, 2989-2992.

17 R. K. Pandey, R. D. Wakharkar and P. Kumar, Synth. Commun., 2005, 35, 2795-2800.

18 G. Cahiez, A. Moyeux and M. Poizat, Chem. Commun., 2014, 50, 8982-8984.

19 P. R. D. Murray, D. L. Browne, J. C. Pastre, C. Butters, D. Guthrie and S. V. Ley, Org. Process Res. Dev., 2013, 17, 1192-1208.

20 T. Stiidemann and P. Knochel, Angew. Chem., Int. Ed. Engl., 1997, 36, 93-95.

21 P. L. Coe and C. E. Scriven, J. Chem. Soc., Perkin Trans. 1, 1986, 475-477.

22 R. B. Miller and M. I. Al-hassan, J. Org. Chem., 1985, 50, 2121-2123.

23 S. D. Brown and R. W. Armstrong, J. Org. Chem., 1997, 62, 7076-7077.

24 D. W. Robertson and J. A. Katzenellenbogen, J. Org. Chem., 1982, 47, 2387-2393.

25 M. Shimizu, C. Nakamaki, K. Shimono, M. Schelper, T. Kurahashi and T. Hiyama, J. Am. Chem. Soc., 2005, 127, 12506-12507.

26 J. Chen, S. Chen, X. Xu, Z. Tang, C.-T. Au and R. Qiu, J. Org. Chem., 2016, 81, 3246-3255.

27 K. Matsumoto and M. Shindo, Adv. Synth. Catal., 2012, 354, 642-650.

28 I. Shiina, M. Suzuki and K. Yokoyama, Tetrahedron Lett., 2004, 45, 965-967.

29 I. Shiina, Y. Sano, K. Nakata, M. Suzuki, T. Yokoyama, A. Sasaki, T. Orikasa, T. Miyamoto, M. Ikekita, Y. Nagahara and Y. Hasome, Bioorg. Med. Chem., 2007, 15, 7599-7617.

30 K. Shimizu, M. Takimoto, M. Mori and Y. Sato, Synlett, 2006, 3182-3184.

31 Y. Takemoto, H. Yoshida and K. Takaki, Chem. - Eur. J., 2012, 18, 14841-14844.

32 Y. Zhou, W. You, K. B. Smith and M. K. Brown, Angew. Chem., Int. Ed., 2014, 53, 3475-3479. 
33 M. Pichette Drapeau, I. Fabre, L. Grimaud, I. Ciofini, T. Ollevier and M. Taillefer, Angew. Chem., Int. Ed., 2015, 54, 10587-10591.

34 Y. Nishihara, M. Miyasaka, M. Okamoto, H. Takahashi, E. Inoue, K. Tanemura and K. Takagi, J. Am. Chem. Soc., 2007, 129, 12634-12635.
35 C. Zhou, D. E. Emrich and R. C. Larock, Org. Lett., 2003, 5, 1579-1582.

36 F. Xue, J. Zhao, T. S. A. Hor and T. Hayashi, J. Am. Chem. Soc., 2015, 137, 3189-3192.

37 C. Zhou and R. C. Larock, J. Org. Chem., 2005, 70, 37653777 . 